\title{
Screening of differentially expressed genes between multiple trauma patients with and without sepsis
}

\author{
S.C. Ji*, Y.T. Pan*, Q.Y. Lu, Z.Y.Sun and Y.Z. Liu \\ Department of Emergency Surgery, Shanghai East Hospital, Shanghai, China \\ *These authors contributed equally to this study. \\ Corresponding authors: Z.Y. Sun / Y.Z. Liu \\ E-mail: sunliu310@hotmail.com
}

Genet. Mol. Res. 13 (1): 1855-1864 (2014)

Received January 3, 2013

Accepted August 10, 2013

Published March 17, 2014

DOI http://dx.doi.org/10.4238/2014.March.17.13

\begin{abstract}
The purpose of this study was to identify critical genes associated with septic multiple trauma by comparing peripheral whole blood samples from multiple trauma patients with and without sepsis. A microarray data set was downloaded from the Gene Expression Omnibus (GEO) database. This data set included 70 samples, 36 from multiple trauma patients with sepsis and 34 from multiple trauma patients without sepsis (as a control set). The data were preprocessed, and differentially expressed genes (DEGs) were then screened for using packages of the $\mathrm{R}$ language. Functional analysis of DEGs was performed with DAVID. Interaction networks were then established for the most up- and downregulated genes using HitPredict. Pathway-enrichment analysis was conducted for genes in the networks using WebGestalt. Fifty-eight DEGs were identified. The expression levels of PLAU (down-regulated) and $M M P 8$ (up-regulated) presented the largest fold-changes, and interaction networks were established for these genes. Further analysis revealed that PLAT (plasminogen activator, tissue) and SERPINF2 (serpin peptidase inhibitor, clade F, member 2), which interact with PLAU, play important roles in the pathway of the component and coagulation cascade. We
\end{abstract}


hypothesize that PLAU is a major regulator of the component and coagulation cascade, and down-regulation of PLAU results in dysfunction of the pathway, causing sepsis.

Key words: Sepsis; Multiple trauma; Differentially expressed genes; Interaction network; Functional enrichment analysis; Pathway analysis

\section{INTRODUCTION}

Multiple trauma occurs when traumas simultaneously occur in 2 or more anatomical sites and when at least 1 trauma is life threatening. Sepsis is a common complication of severe traumas, burns, shocks, major surgeries, and infections (Rice and Bernard, 2005). If sepsis is not effectively controlled at an early stage, septic shock and multiple organ dysfunction syndrome will occur, causing a high mortality rate (Taylor et al., 1991). Statistical data show that 70 million people suffer from sepsis in the United States each year, of which 21 million people die, corresponding to a mortality rate of $30 \%$. This number is greater in China, approximately 45\% (Riedemann et al., 2003). Wafaisade et al. (2011) showed that although the incidence of sepsis has decreased in Germany, there has been no significant decrease in mortality in the subgroup of septic trauma patients. Considering the danger and mortality of sepsis, it is urgent to characterize the deteriorative process and then develop effective treatments.

Researchers have adopted various methods to uncover different aspects of the underlying mechanisms of sepsis. Studies (Chen et al., 2011; Zhao et al., 2012) have linked polymorphisms in the Toll-like receptor 9-encoding gene to sepsis and multiple organ dysfunction. Polymorphisms of the tumor necrosis factor-encoding gene (Majetschak et al., 2002; O'Keefe et al., 2002) and interferon-gamma-encoding gene (Stassen et al., 2002) have also been investigated. Gouel-Cheron et al. (2012) found that a high interleukin-6 (IL-6) concentration and a persistent decrease in monocytic human leukocyte antigen DR (mHLA-DR) expression are indicators for development of sepsis. Microarray technology allows a widely used gene expression profile method for exploring molecular mechanisms. Using this method, Paunel-Gorgulu et al. (2012) explored the delayed apoptosis in neutrophils from multiple trauma patients with and without sepsis.

Considerable advancements have been made in the study sepsis, especially elucidating potential biomarkers, such as c-type natriuretic peptide (Suttner and Boldt, 2010), soluble Fas (Paunel-Gorgulu et al., 2011), procalcitonin (Svoboda et al., 2007), and IL-6 (Haasper et al., 2010). Several therapies have also been proposed, e.g., estrogen treatment (Raju and Chaudry, 2008; Kawasaki and Chaudry, 2012). However, knowledge about sepsis is inadequate for achieving effectively clinical control. Therefore, we compared multiple trauma patients with and without sepsis by microarray technology to identify key genes implicated in the process, which have the potential to be used as the targets to combat the disease.

\section{MATERIAL AND METHODS}

\section{Microarray data}

Data set GSE12624 was downloaded from the Gene Expression Omnibus (GEO) database, comprising 70 peripheral blood samples, 36 from multiple trauma patients with sepsis 
and 34 from multiple trauma patients without sepsis. The microarray data had been collected using a GPL4204 GE Healthcare/Amersham Biosciences CodeLinkUniSet Human I Bioarray instrument. Raw data were downloaded along with the probe annotation files.

\section{Pre-processed data and differentially expressed genes (DEGs) identification}

Data were pre-processed using Affy (Troyanskaya et al., 2001; Fujita et al., 2006) and part of the R language. Differential expression analysis was performed using Limma package (Smyth, 2005). The Bayesian method (Benjamini and Hochberg, 1995) was employed for multiple testing corrections. A false-discovery rate (FDR) $<0.05$ and $|\operatorname{logFC}|>1$ were the cutoffs to filter DEGs. Genes with the maximum fold-changes (FC) in expression levels were targeted for deep mining.

\section{Functional analysis of all DEGs}

Functional analysis was performed for all of the DEGs using the Expressing Analysis Systematic Explorer (EASE) (Hosack et al., 2003). FDR $<0.05$ was regarded as significant.

\section{Establishing protein-protein interaction networks}

Because most proteins function in concert or in complexes to exerting their biological functions, building interaction networks is beneficial for understanding of protein functions. HitPredict was utilized to establish the interaction networks for protein products of the most up- and down-regulated genes.

HitPredict includes protein-protein interactions from the IntAct, BIOGRID, and HPRD databases; information was originally obtained from high-throughput or small-scale experiments. HitPredict also contains predicted interactions based on scores that integrate information such as sequence, structure, and function using a Bayesian algorithm (Patil et al., 2011). Interactions obtained from experiments or with a score $>1$ are likely of significance (Patil and Nakamura, 2005) and form a basis for our interaction networks.

\section{Pathway-enrichment analysis of network}

WebGestalt (Zhang et al., 2005; Duncan et al., 2010) was employed to analyze the genes in the networks, using a hypergeometric distribution algorithm to uncover the functions, and adj. $\mathrm{P}<0.05$ was set as the cutoff.

\section{RESULTS}

\section{Differentially expressed genes}

Microarray data were preprocessed and normalized (Figure 1). Fifty-eight DEGs were identified according to the criteria $(\mathrm{P}<0.05$ and $|\operatorname{logFC}|>1)$ : 21 down-regulated and 37 upregulated DEGs (Table 1). PLAU ( $\operatorname{logFC}=-1.32793955)$ was the most down-regulated gene, while MMP8 $(\log \mathrm{FC}=1.74640401)$ was the most up-regulated gene (Figure 2$)$. These two genes were selected for further analysis. 


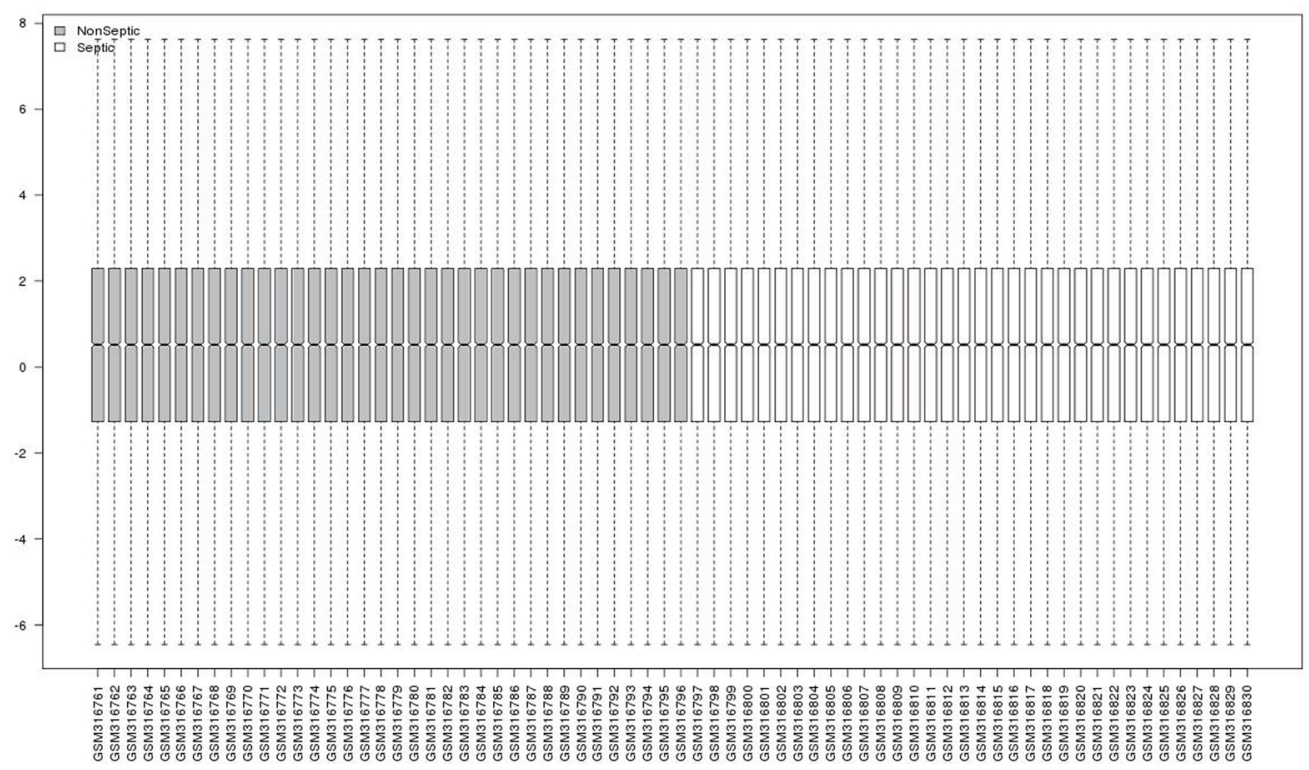

Figure 1. Box plot for normalized gene expression data, 34 gray boxes for non-sepsis-induced multiple trauma samples and 36 white boxes for sepsis-induced multiple trauma samples. Black line in the box represents the median of data set. The black lines are almost on the same line, indicating good effectiveness of normalization.

Table 1. List for differentially expressed genes.

\begin{tabular}{|c|c|c|c|c|c|}
\hline Gene symbol & FDR & $\log \mathrm{FC}$ & Gene symbol & FDR & $\log \mathrm{FC}$ \\
\hline PLAU & $2.65 \mathrm{E}-05$ & -1.32794 & ITGA7 & 0.003925 & 1.047575 \\
\hline $\mathrm{ABP} 1$ & 0.000767 & -1.31794 & SAMSN1 & 0.000543 & 1.054721 \\
\hline KIAA0484 & 0.002364 & -1.30972 & KLHL2 & 0.002387 & 1.07438 \\
\hline CPA3 & 0.018433 & -1.29321 & IPO11 & $6.1 \mathrm{E}-07$ & 1.075049 \\
\hline SPP1 & 0.009126 & -1.25878 & HAT1 & $2.65 \mathrm{E}-05$ & 1.093273 \\
\hline HSD17B3 & 0.000799 & -1.24959 & TPST1 & 0.014875 & 1.10131 \\
\hline RPS6KA5 & 0.000549 & -1.22299 & CLEC2B & 0.000934 & 1.102343 \\
\hline ETV7 & 0.028783 & -1.22112 & C9orf103 & 0.000129 & 1.106543 \\
\hline SPON2 & 0.000672 & -1.20473 & DHRS9 & 0.000864 & 1.159279 \\
\hline MPZL2 & 0.000119 & -1.20289 & BIRC5 & 0.00049 & 1.161581 \\
\hline EXOSC5 & 0.028183 & -1.18981 & CPNE5 & 0.000919 & 1.163124 \\
\hline SLC45A4 & 0.008763 & -1.13275 & VSIG4 & 0.010235 & 1.164657 \\
\hline GNLY & $6.44 \mathrm{E}-05$ & -1.1292 & UQCRB & 0.000515 & 1.168334 \\
\hline PLA2G7 & 0.003009 & -1.0861 & PROS1 & $2.65 \mathrm{E}-05$ & 1.177566 \\
\hline ZNF12 & 0.002486 & -1.04606 & GUCY1B3 & $3.31 \mathrm{E}-05$ & 1.218599 \\
\hline SERPINB2 & 0.017122 & -1.04274 & TNFRSF17 & $7.9 \mathrm{E}-05$ & 1.239881 \\
\hline MME & 0.00056 & -1.03697 & PRUNE2 & $2.65 \mathrm{E}-05$ & 1.273587 \\
\hline APCDD1 & 0.005063 & -1.02828 & OLAH & 0.000396 & 1.319922 \\
\hline GZMH & 0.000209 & -1.0275 & MED18 & 0.001043 & 1.326346 \\
\hline XAF1 & 0.008767 & -1.02284 & CWF19L1 & 0.009126 & 1.334516 \\
\hline EPHB4 & $6.51 \mathrm{E}-05$ & -1.0209 & ARG1 & 0.000228 & 1.392289 \\
\hline PDZD8 & 0.0002 & 1.005948 & EDG1 & 2.39E-06 & 1.416898 \\
\hline ERLIN1 & 0.005074 & 1.009205 & IGJ & 0.000767 & 1.420944 \\
\hline BAZ1A & 0.004154 & 1.015047 & TACSTD1 & 0.048965 & 1.455898 \\
\hline LEPROT & 0.00032 & 1.018187 & PCOLCE2 & $2.65 \mathrm{E}-05$ & 1.475443 \\
\hline PCSK1 & 0.006772 & 1.0192 & C5orf30 & 0.003405 & 1.557862 \\
\hline SYCP2 & $6.51 \mathrm{E}-05$ & 1.030233 & WFDC1 & 0.01506 & 1.618033 \\
\hline RNASE1 & 0.016689 & 1.036442 & HPGD & 0.001807 & 1.732723 \\
\hline SMPDL3A & 0.012126 & 1.038862 & MMP8 & 0.008898 & 1.746404 \\
\hline
\end{tabular}

$\mathrm{FDR}=$ false-discovery rate $\mathrm{FC}=$ fold-changes. 


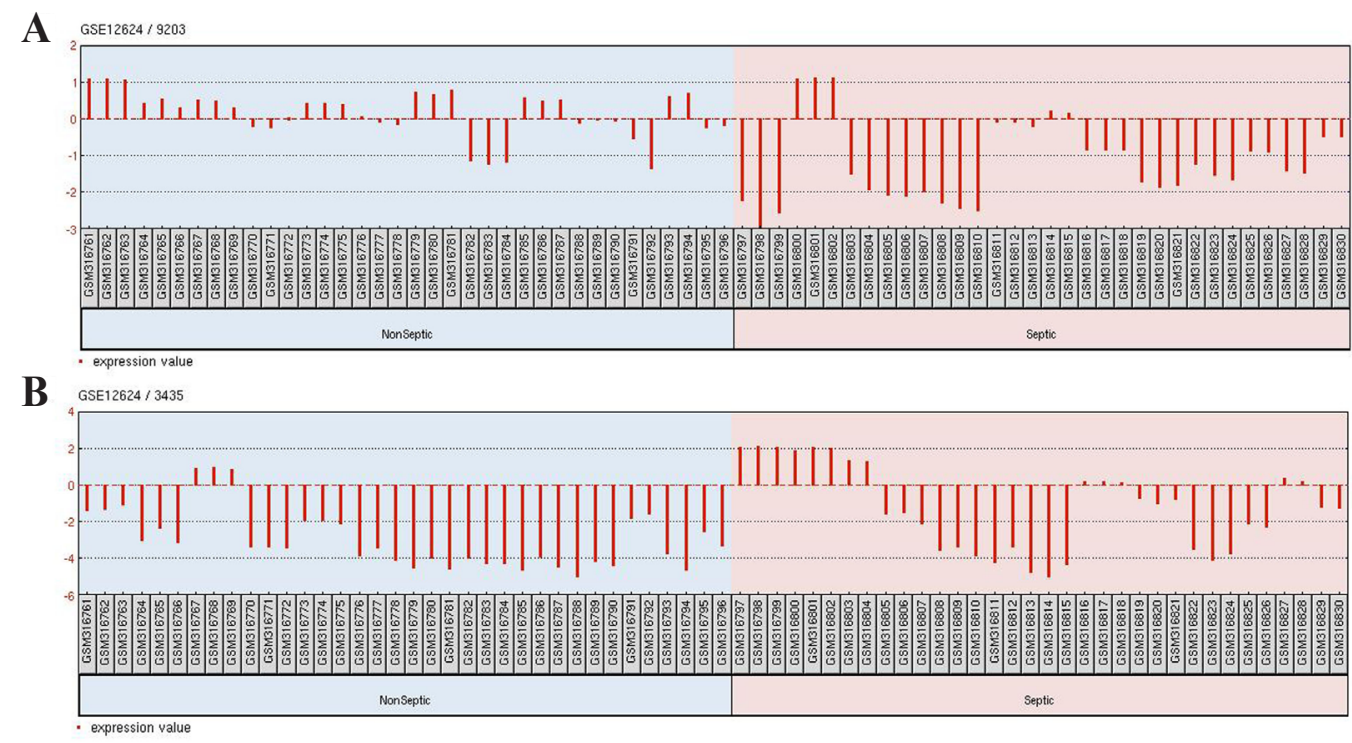

Figure 2. Expression data of down-regulated $P L A U(\mathbf{A})$ and up-regulated $M M P 8(\mathbf{B})$ for all the samples.

\section{Functional analysis of all DEGs}

Functional analysis of the DEGs revealed 3 significantly enriched terms (Table 2), among which GO: 0009611 - response to wounding - was the most significant term. Eight DEGs were associated with this function, TPST1, PCSK1, SERPINB2, PLA2G7, VSIG4, $P R O S 1, P L A U$, and SPP1. The term of proteolysis was also enriched.

Table 2. Functional analysis results for all of the differentially expressed genes.

\begin{tabular}{lll}
\hline Term & \multicolumn{1}{c}{ Adj. P } & Genes \\
\hline GO:0009611 - response to wounding & 0.00148 & TPST1, PCSK1, SERPINB2, PLA2G7, VSIG4, PROS1, PLAU, SPP1 \\
GO:0006508 - proteolysis & 0.018919 & RPS6KA5, PCSK1, MMP8, MME, CPA3, ERLIN1, GZMH, VSIG4, PLAU \\
GO:0007565 - female pregnancy & 0.0496789 & HPGD, PLAU, SPP1 \\
\hline
\end{tabular}

\section{Further analysis of PLAU and MMP8}

Interaction networks for the protein products of $P L A U$ and $M M P 8$ were comprised of 19 and 7 proteins, respectively (Figure 3). Pathway-enrichment analysis revealed that proteins in the former network enriched in ko04610: complement and coagulation cascade (Figure 4). PLAT and SERPINB2 were involved in this pathway.

\section{DISCUSSION}

We compared gene expression data from multiple trauma patients with and without sepsis and identified 58 DEGs. Functional analysis found 3 significant terms with apparent or unapparent associations with the disease. We further analyzed the most down-regulated gene 


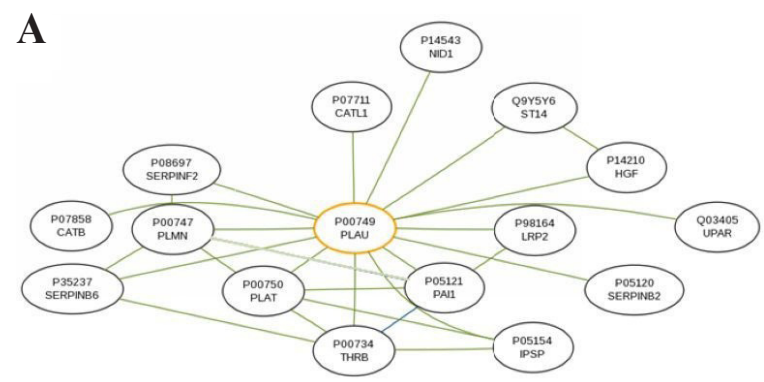

\section{B}

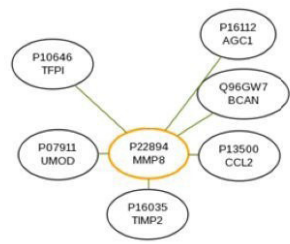

Interaction Edge Legend

- High confidence

.....- Spurious small-scale or high-throughput

Figure 3. Interaction networks for PLAU (A) and MMP8 (B) generated by HitPredict. Green line represents a connection of high confidence (likelihood $>1$ ).

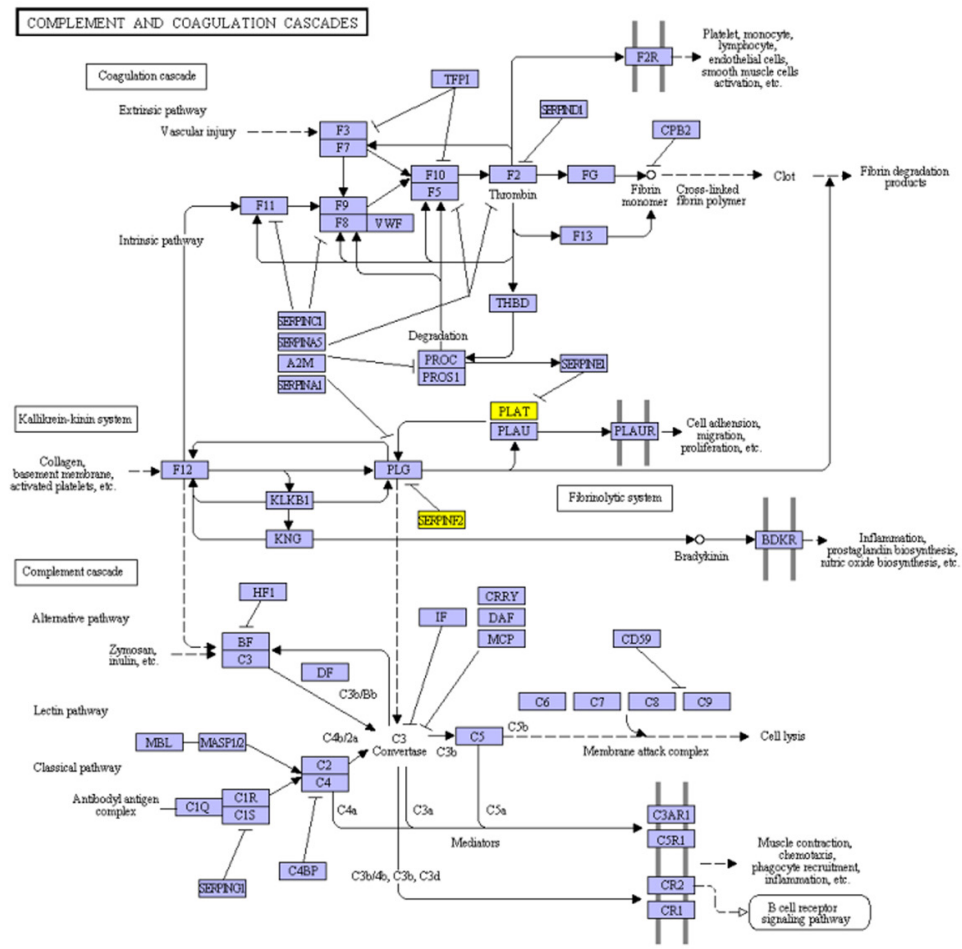

Figure 4. Schematic diagram for complement and coagulation cascades. The yellow box indicates protein that interacts with PLAU. 
$P L A U$ and the most up-regulated gene MMA8. Eighteen and six interaction partners for PLAU and $M M A 8$, respectively, were acquired using bioinformatic tools, and the functions of the interaction partners were explored (Table 3 ). The pathway of the complementary and coagulation cascade was identified for the former group of interaction partners.

\begin{tabular}{llc}
\multicolumn{2}{l}{ Table 3. Interactors for PLAU (A) and MMP8 (B). } & \\
\hline A & & Likelihood score \\
\hline ID & Interactor & 999 \\
\hline 160779 & SERPINB6 & 999 \\
160800 & PLMN & 999 \\
160822 & PAI1 & 999 \\
160828 & PLAT & 999 \\
160839 & SERPINB2 & 999 \\
160842 & UPAR & 999 \\
161280 & THRB & 999 \\
163183 & LRP2 & 999 \\
163185 & IPSP & 999 \\
163186 & ST14 & 999 \\
169965 & CATB & 999 \\
170178 & CATL1 & 999 \\
170456 & SERPINF2 & 999 \\
174248 & HGF & 999 \\
179105 & NID1 & 999 \\
180745 & MPRI & 999 \\
214893 & SERPINE2 & 3.37 \\
\hline 195271 & BTK & Likelihood score \\
\hline B & & 999 \\
\hline ID & Interactor & 999 \\
\hline 173399 & CCL2 & 999 \\
173402 & TIMP2 & 999 \\
173403 & BCAN & 999 \\
173405 & UMOD & 999 \\
176642 & TFPI & \\
\hline 17672 & AGC1 & \\
\hline
\end{tabular}

The most significant function term was response to wounding, which was expected because multiple trauma had occurred to the patient. Eight proteins belong to this group. The changes in expression levels of several genes may provide an explanation for the incidence of sepsis. V-set and immunoglobulin domain containing 4 (VSIG4) functions as a negative regulator of T cell activation (Vogt et al., 2006) but VSIG4 is up-regulated in sepsis patients. In contrast, secreted phosphoprotein 1 (SPP1), a cytokine that up-regulates expression of interferon-gamma and IL-12, is down-regulated in sepsis patients. Plasminogen activator inhibitor-2 (SerpinB2), which inhibits the proteasome (Boncela et al., 2011), is down-regulated in our study, favoring protein degradation in accordance with the second function term, proteolysis. However, Schroder et al. (2010) reported that SerpinB2 could play a positive role in adaptive immunity. Further studies on SerpinB2 polymorphisms may be beneficial and interesting. In addition, protein $\mathrm{S}$ (alpha) (PROS1) is up-regulated and is a cofactor for the anticoagulant protease.

Because accelerated proteolysis of muscle is characteristic of patients with sepsis (Clowes Jr. et al., 1983), the term of proteolysis is also significantly enriched. Several DEGs associated with degradation of the extracellular matrix were included in this group, but these 
DEGs presented different changes in the expression level. The protein encoded by the most up-regulated gene $M M A 8$ was included in this group. MMA8 is a member of the matrix metalloproteinase (MMP) family that is involved in the breakdown of the extracellular matrix. Plasminogen activator urokinase (PLAU) is a serine protease involved in degradation of the extracellular matrix and its gene is down-regulated in patients with sepsis. In addition, granzyme $\mathrm{H}(\mathrm{GZMH})$, which plays a role in innate immune response, is down-regulated at the gene expression level. This observation is in accordance with previous research on granzyme A (Accardo-Palumbo et al., 2010).

Our analysis also suggested that complement and coagulation cascades were implicated in the pathological process of sepsis. Previous studies have shown that an overactive complement system induced by sepsis is harmful (Ward et al., 2003; Albrecht and Ward, 2005) and may even cause multiple organ failure (Kansas, 1996; Flierl et al., 2006). Schefold et al. (2007) performed an interventional extracorporeal treatment that simultaneously reduced the levels of endotoxin, IL-6, and C5a via selective extracorporeal immunoadsorption, and they successfully restored monocytic responsiveness and improved organ function. Janssen et al. (2007) investigated the efficacy of complement inhibitor - compstatin - on complement component $\mathrm{C} 3$ and the treatment of sepsis using a baboon sepsis model. They find that compstatin could lower the activity of complement in plasma and tissue, reduce the accumulation of macrophages and platelets in organs, and decrease the clotting activity through down-regulation of coagulation factors I and plasminogen activator inhibitor-1. Therefore, inhibition of the undesired activity of the complement system can be a promising means of protecting organ function.

Because PLAU interacts with PLAT and SerpinB2, which are involved in the complement cascade, we speculate that PLAU is a regulator for normal function of the complement system, and the down-regulation of PLAU may contribute to the dysfunction of the system that subsequently causes multiple organ injury in patients.

In summary, we discovered several important DEGs likely involved in the pathological process of sepsis. These DEGs may represent potential drug targets for multiple trauma patients with sepsis. Nevertheless, more research is necessary to study the function and role of these DEGs in sepsis.

\section{REFERENCES}

Accardo-Palumbo A, D'Amelio L, Pileri D, D'Arpa N, et al. (2010). Reduction of plasma granzyme A correlates with severity of sepsis in burn patients. Burns 36: 811-818.

Albrecht EA and Ward PA (2005). Complement-induced impairment of the innate immune system during sepsis. Curr. Infect. Dis. Rep. 7: 349-354.

Benjamini Y and Hochberg Y (1995). Controlling the false discovery rate: A practical and powerful approach to multiple testing. J. R. Stat. Soc. B 57: 289-300.

Boncela J, Przygodzka P, Papiewska-Pajak I, Wyroba E, et al. (2011). Association of plasminogen activator inhibitor type 2 (PAI-2) with proteasome within endothelial cells activated with inflammatory stimuli. J. Biol. Chem. 286: 43164-43171.

Chen KH, Zeng L, Gu W, Zhou J, et al. (2011). Polymorphisms in the Toll-like receptor 9 gene associated with sepsis and multiple organ dysfunction after major blunt trauma. Br. J. Surg. 98: 1252-1259.

Clowes GH Jr, George BC, Villee CA Jr and Saravis CA (1983). Muscle proteolysis induced by a circulating peptide in patients with sepsis or trauma. N. Engl. J. Med. 308: 545-552.

Duncan D, Prodduturi N and Zhang B (2010). WebGestalt2: an updated and expanded version of the Web-based Gene Set Analysis Toolkit. BMC Bioinformatics 11: 10. 
Flierl MA, Schreiber H and Huber-Lang MS (2006). The role of complement, C5a and its receptors in sepsis and multiorgan dysfunction syndrome. J. Invest. Surg. 19: 255-265.

Fujita A, Sato JR, Rodrigues LO, Ferreira CE, et al. (2006). Evaluating different methods of microarray data normalization. BMC Bioinformatics 7: 469.

Gouel-Cheron A, Allaouchiche B, Guignant C, Davin F, et al. (2012). Early interleukin-6 and slope of monocyte human leukocyte antigen-DR: a powerful association to predict the development of sepsis after major trauma. PLoS One 7: e33095.

Haasper C, Kalmbach M, Dikos GD, Meller R, et al. (2010). Prognostic value of procalcitonin (PCT) and/or interleukin-6 (IL-6) plasma levels after multiple trauma for the development of multi organ dysfunction syndrome (MODS) or sepsis. Technol. Health Care 18: 89-100.

Hosack DA, Dennis G Jr, Sherman BT, Lane HC, et al. (2003). Identifying biological themes within lists of genes with EASE. Genome Biol. 4: R70.

Janssen BJ, Halff EF, Lambris JD and Gros P (2007). Structure of compstatin in complex with complement component C3c reveals a new mechanism of complement inhibition. J. Biol. Chem. 282: 29241-29247.

Kansas GS (1996). Selectins and their ligands: current concepts and controversies. Blood 88: 3259-3287.

Kawasaki T and Chaudry IH (2012). The effects of estrogen on various organs: therapeutic approach for sepsis, trauma, and reperfusion injury. Part 2: liver, intestine, spleen, and kidney. J. Anesth. 26: 892-899.

Majetschak M, Obertacke U, Schade FU, Bardenheuer M, et al. (2002). Tumor necrosis factor gene polymorphisms, leukocyte function, and sepsis susceptibility in blunt trauma patients. Clin. Diagn. Lab. Immunol. 9: 1205-1211.

O'Keefe GE, Hybki DL and Munford RS (2002). The G $\rightarrow$ A single nucleotide polymorphism at the -308 position in the tumor necrosis factor-alpha promoter increases the risk for severe sepsis after trauma. J. Trauma 52: 817-825.

Patil A and Nakamura H (2005). Filtering high-throughput protein-protein interaction data using a combination of genomic features. BMC Bioinformatics 6: 100.

Patil A, Nakai K and Nakamura H (2011). HitPredict: a database of quality assessed protein-protein interactions in nine species. Nucleic Acids Res. 39: D744-D749.

Paunel-Gorgulu A, Flohe S, Scholz M, Windolf J, et al. (2011). Increased serum soluble Fas after major trauma is associated with delayed neutrophil apoptosis and development of sepsis. Crit. Care 15: R20.

Paunel-Gorgulu A, Kirichevska T, Logters T, Windolf J, et al. (2012). Molecular mechanisms underlying delayed apoptosis in neutrophils from multiple trauma patients with and without sepsis. Mol. Med. 18: 325-335.

Raju R and Chaudry IH (2008). Sex steroids/receptor antagonist: their use as adjuncts after trauma-hemorrhage for improving immune/cardiovascular responses and for decreasing mortality from subsequent sepsis. Anesth. Analg. 107: 159-166.

Rice TW and Bernard GR (2005). Therapeutic intervention and targets for sepsis. Annu. Rev. Med. 56: 225-248.

Riedemann NC, Guo RF and Ward PA (2003). Novel strategies for the treatment of sepsis. Nat. Med. 9: 517-524.

Schefold JC, von Haehling S, Corsepius M, Pohle C, et al. (2007). A novel selective extracorporeal intervention in sepsis: immunoadsorption of endotoxin, interleukin 6, and complement-activating product 5a. Shock 28: 418-425.

Schroder WA, Le TT, Major L, Street S, et al. (2010). A physiological function of inflammation-associated SerpinB2 is regulation of adaptive immunity. J. Immunol. 184: 2663-2670.

Smyth GK (2005). Limma: Linear Models for Microarray Data. In: Bioinformatics and Computational Biology Solutions using R and Bioconductor (Gentleman R, Carey V, Dudoit S, Irizarry R, et al., eds.). Springer, New York, 397-420.

Stassen NA, Leslie-Norfleet LA, Robertson AM, Eichenberger MR, et al. (2002). Interferon-gamma gene polymorphisms and the development of sepsis in patients with trauma. Surgery 132: 289-292.

Suttner S and Boldt J (2010). Prediction of sepsis after multiple trauma: does C-type natriuretic peptide do the trick? Crit. Care Med. 38: 323-324.

Svoboda P, Kantorova I, Scheer P, Radvanova J, et al. (2007). Can procalcitonin help us in timing of re-intervention in septic patients after multiple trauma or major surgery? Hepatogastroenterology 54: 359-363.

Taylor FB Jr, Chang A, Ruf W, Morrissey JH, et al. (1991). Lethal E. coli septic shock is prevented by blocking tissue factor with monoclonal antibody. Circ. Shock 33: 127-134.

Troyanskaya O, Cantor M, Sherlock G, Brown P, et al. (2001). Missing value estimation methods for DNA microarrays. Bioinformatics 17: 520-525.

Vogt L, Schmitz N, Kurrer MO, Bauer M, et al. (2006). VSIG4, a B7 family-related protein, is a negative regulator of T cell activation. J. Clin. Invest. 116: 2817-2826.

Wafaisade A, Lefering R, Bouillon B, Sakka SG, et al. (2011). Epidemiology and risk factors of sepsis after multiple trauma: an analysis of 29,829 patients from the Trauma Registry of the German Society for Trauma Surgery. Crit. Care Med. 39: 621-628. 
Ward PA, Riedemann NC, Guo RF, Huber-Lang M, et al. (2003). Anti-complement strategies in experimental sepsis. Scand. J. Infect. Dis. 35: 601-603.

Zhang B, Kirov S and Snoddy J (2005). WebGestalt: an integrated system for exploring gene sets in various biological contexts. Nucleic Acids Res. 33: W741-W748.

Zhao P, Lu G and Cai L (2012). Polymorphisms in the Toll-like receptor 9 gene associated with sepsis and multiple organ dysfunction after major blunt trauma (Br. J. Surg. 98: 1252-1259). Br. J. Surg. 99: 145 (author reply). 